\title{
The Potential Opportunity of Promoting Meituan Take-out App to Hong Kong - A Case Study
}

\author{
Li Shen \\ Business School, Lingnan University, Hong Kong, China
}

Email address:

1713983053@qq.com

To cite this article:

Li Shen. The Potential Opportunity of Promoting Meituan Take-out App to Hong Kong - A Case Study. International Journal of Economic Behavior and Organization. Vol. 6, No. 2, 2018, pp. 53-60. doi: 10.11648/j.ijebo.20180602.14

Received: November 5, 2018; Accepted: November 26, 2018; Published: December 11, 2018

\begin{abstract}
This paper presents a case study of proposing a marketing plan for Meituan Take-out App. This take-out app is quite popular in mainland area now and experienced a quick development over past several years under the Internet era. With the increase of scale and strength rapidly, it faces a potential opportunity to enter into the Hong Kong market. Thus the paper makes a corresponding research under the assumption that it develops the local market in Hong Kong. The aim of this study is to explore the prospects of its development in the Hong Kong market and then put forward an effective and reasonable promotion plan. In order to promote this take-out app, a detailed analysis is formulated based on both consumer buying process and consumer evaluation process, as well as considering the target audience and market positioning of this brand. In addition, some well-known marketing theories and research methods like PEST analysis are also applied for sake of achieving better research results. At last, useful recommendations are provided in the end of paper, to ensure the smooth application of proposed promotion in Hong Kong. It is hoped that this case study could serve as reference and guidance for Meituan Take-out App and other similar companies.
\end{abstract}

Keywords: Consumer Buying, Consumer Evaluation, Marketing, Case Study, Hong Kong

\section{Introduction}

Meituan Take-out Application is an online ordering platform, which is under Meituan Group. The business model of Meituan Take-out application is "online to offline" which is used in digital marketing to describe systems enticing consumers within a digital environment to make purchases of goods or services from physical businesses.

The Meituan Take-out Application is launched at the end of 2013. After five-years rapid development, Meituan becomes one of the largest online ordering platforms in the world. It has more than 2 million business users and over 250 million personal users from over 300 cities of Mainland China. Meituan take-out application enables to receive 18 million orders per day. And over 500,000 takeout deliverymen are responsible for the delivery service. In 2017, the total transactions had reached 171 billion.

Actually, it is necessary to mention that there are four major ways for Hong Kong citizens to draw on take-away services. The first way is self pick-up the food from the restaurant. To a certain extent, it is convenient for the restaurant; However, self pick-up food is extremely inconvenient for consumers who spend long time in standing in a queue, especially at peak hours. The second way is that ordering takeout by phone, which consumers may encounter a bad user experience. This is because the line consumer dialed is busy during peak hours. And consumers are not able to browse the full menu and then choose the meals what they prefer. The third way is that ordering online on the official website. In fact, the employees of certain large-scale food and beverage enterprises like McDonald, KFC, Pizza Hut and local restaurant, undertake delivery services. There are few restaurants with delivery service for customers to choose. At peak hours, the unbelievable long time is owing to the insufficiency of delivery guys. The eventual way that pick-up by colleagues or friends that no one would like to do in long term.

Currently, there are three take-out apps in Hong Kong: Foodpanda, Deliveroo and Uber Eats. Foodpanda and Deliveroo are two most popular applications.

The objective of report is concerning Meituan take-out application's potential opportunity in Hong Kong market, thus the priority of the project is to view mainland students from eight universities of Hong Kong as target customers. The aim of this paper is to analyze the promotion prospects of Meituan 
take-out app in HK. Thus the paper will demonstrate the consumer buying process analysis that consists of consumer needs and buying process, and the consumer evaluation process analysis which includes PEST and SWOT analysis of the brand. Then the product focus and goal setting will be shown. The recommendations for marketing actions will also be provided as well.

\section{Consumer Buying Process Analysis}

\subsection{Consumer Need Analysis}

Prior to promoting Meituan App to Hong Kong university students who are from Mainland China, comprehending consumer need is instrumental in seizing the opportunity and grabbing a large number of market share in the future. Hence, it is inevitable to highlight the superiority of Meituan Take-out app in comparison to the existing take-out apps in Hong Kong. In other words, the emergence of negative comments means the services that cannot meet consumer satisfaction and expectation. Based on the analysis on the current background of take-out service and the main competitors in Hong Kong, Meituan App ought to do a market research on consumer needs [1].

\subsubsection{Maslow's Hierarchy of Needs}

Theoretically, the self-esteem of consumers [2] can be achieved. In other words, the convenient need is acquired by delivery services of Meituan App. In comparison to share a table with others or queue up for a meal, the consumers will generate a sense of being respect through the delivery services provided by Meituan App. Still, consumers' belonging need can be satisfied. Concerning the taste or flavour of food, the mainland students incline to find their self-identity group, because their hometown is far away from Hong Kong. Psychologically, the sense of affability may be increased by the satisfaction towards the hometown dishes. And the hometown-flavour dishes are capable to deploy the homesickness.

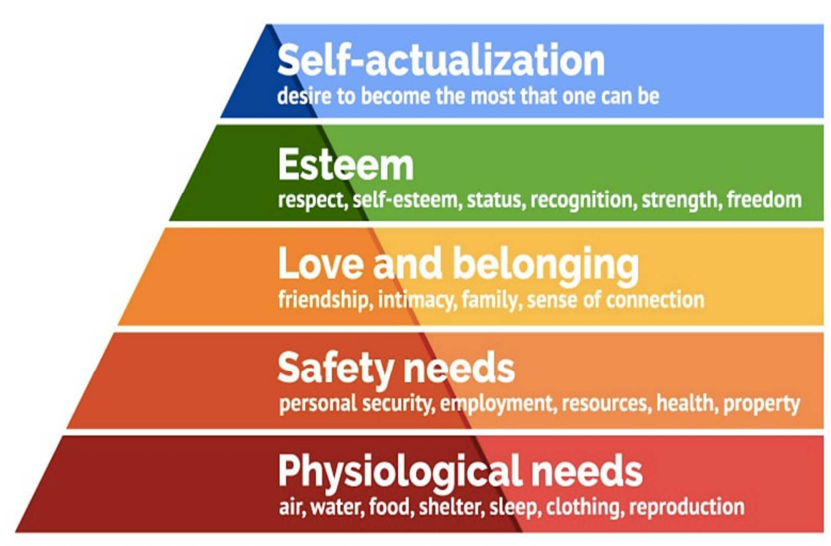

Figure 1. Maslow's Hierarchy of Needs.

\subsubsection{Specific Needs and Buying Behaviour}

From the matrix of specific needs and buying behaviour [3], the need for power can be applied. Consumers suppose that they are able to control their environment and unwilling to share a table with others. By coincidence, Meituan take-out app is able to provide the food delivery service to such type of consumers.

\subsection{Buying Process Analysis}

\subsubsection{The Prospect of Mobile Payment}

The target consumers are Hong Kong university students from mainland China when introducing Meituan to Hong Kong. If the business condition is in line with expectation, the next target consumers will be expanded. For attracting consumers to use Meituan take-out app, it is very important to make a forecast for the prospect of mobile payment in Hong Kong [4].

Meituan take-out app [5] is an Internet ordering platform, which is based on the location based service (LBS). The app is a product of the combination of $\mathrm{O} 2 \mathrm{O}$ and the take-out industry. Hence, it is vitally essential to comprehend the prospect of mobile payment in Hong Kong. Initially, consumers ought to finish payment online, it is inevitable to mention the mobile penetration rate in Hong Kong. A research report indicated that $96 \%$ smartphone users in Hong Kong use their smartphone to go online every day, the figure is highest in Asia Pacific. And 40.9\% smartphone owners are addicted to online shopping. Still, a survey disclosed that almost $90 \%$ male and $80 \%$ female possess smartphone, and the main age groups range from teens to adults. Importantly, the mobile penetration rate reached $239 \%$, which ranked highest in the world.

Secondly, it is necessary to mention the existing payment mode and the prospect for new mobile payment in Hong Kong. In addition to cash and card payment, WeChat Pay HK and Alipay HK currently are the burgeoning payment ways with a series of attractive discounts and random reductions. Concerning Alipay HK, Alipay launched the Hong Kong version of the e-wallet. Alipay HK can bind the local bank's credit card. Hong Kong residents are able to recharge the balance or pay directly in Hong Kong dollars by Alipay HK. On March 8, 2018, Alipay HK had more than 1 million users in Hong Kong and more than 10,000 cooperative merchants. Regarding WeChat Pay HK, the quick payment QR code is designed for restaurants, supermarkets, chains stores and other merchants. Merchants can connect WeChat payment HK to the retail system of POS machines to directly complete the charges. The QR code collection is designed for small businesses such as individuals, street shops, and home-style shops. No POS machines need to be installed. Consumers can use the WeChat scan code to complete payment directly.

\subsubsection{Top Pain Points of Catering Delivery Service in HK}

Currently, there are certain problems that have led to low interest for online ordering meals by apps. The Euro-monitor research indicated that the gross income of catering industry reached $\$ 650$ million in 2016; however, the catering delivery service only accounted for $3 \%$ of general income ( $\$ 20$ million). Surprisingly, Hong Kong citizens mainly order takeaway by phone or take meals by friends. Some of them 
may grab their meals by themselves because of no seats provided by restaurant at peak hours.

Actually, before introducing Meituan app to $\mathrm{HK}$, the company is confronted with the current pain points in HK's catering delivery service. The first dilemma is that the narrow road and mountainous terrain may affect the order quantity. However, the dilemma will not be difficult for Meituan to overcome. Meituan takeout platform performed well in Chongqing where is a famous mountainous city in Mainland China. In 2017, The Business Forum of Meituan Takeout Merchants issued a fact that the ordering quantity of Meituan in Chongqing was quadrupled last year. The second dilemma is that the hefty price tag on human cost may aggravate the harshness of hiring. The third dilemma is that paying by cash or card (PayPal) is the dominant payment mode. The forth dilemma is that the confine of the existing apps, which is Uber Eats. In other words, in the course of ordering meals by Uber Eats, consumers found that they can select the meals by such platform; however, the location is not within the delivery coverage, leading to the failure of the transaction.

\subsubsection{Consumer Purchasing Decision}

The promotion of the app is different from the specific product. The initial stage of application is the stage that requires high investment, while the initial stage of product promotion is the stage of low investment. This is the basic difference between the two in the promotion. Therefore, if the Meituan take-out app expects to develop in Hong Kong, it is inevitable to analyze consumer buying process model [6] before introducing.

a) Problem recognition (see appendix)

At present, there are a total of eight public universities in Hong Kong. The total number of students is more than 110,000 . During the peak period of school restaurants, the supply of goods often falls short of demand. However, the existing take-away APP in the Hong Kong market cannot meet the needs of students. This time, it needs a better product to enter the market.

So it is required to design a questionnaire to conduct the survey. The main questions on the questionnaire are: Do students in Hong Kong need to use takeout APP? What kind of takeout APP is required for HK students? What kind of services is provided for $\mathrm{HK}$ students?

b) Evaluation of alternatives (homogenous analysis [7])

The following three types of take-away Apps are available in the Hong Kong market, such as Foodpanda, Deliveroo, and Uber Eats. Based on the respective characteristics of competitors, the homogenous analysis of are shown as follow:

Foodpanda is the first app to launch in Hong Kong and currently is the largest delivery platform in Hong Kong. There are more than 1,000 cooperative restaurants, some of which are high quality restaurants. The payment method offers cash on delivery, PayPal and online payment, consumers can track the delivery route through an external map, and some restaurants will cooperate with Foodpanda to avoid delivery fees.

Deliveroo is an app that cooperates with large groups, such as Maxim Group, Swire Group, etc. The scope of services covers most of the nine major areas in Hong Kong. Only the old and famous restaurants, the other restaurants recommended by Michelin can be delivered. In the Kwun Tong area, you can also take delivery of the takeaways from Wahsou Icehouse. The Payment method provides apple pay or credit card, but the delivery fee is needed. If the order totally is less than $120 \mathrm{HKD}$, an additional $55 \mathrm{HKD}$ is required; if there is more than $120 \mathrm{HKD}$, a $20 \mathrm{HKD}$ service fee will be charged.

Uber Eats: The co-operate merchants are most authentic restaurants, local restaurants like Longhua Hotel, Sheng Ke and so on. The poster of Uber Eats is full of emotion and sincere. However, the problem is that many customers find that the area where he living is not in the delivery range.

Therefore, Meituan should avoid the disadvantages of rivals and use the advantages of competitors. For instance, the app ought to cover the main area of residence and minimize the cost of delivery fee while enriching payment methods, and cooperate with the most popular restaurants as much as possible.

c) Purchase decision (see appendix)

Consumers will form purchase intentions through comparison with other apps. At this time, it will be use of the Meituan's good reputation among domestic consumers and the big data accumulated by the Meituan over a long period of time will enable consumers to make purchase decisions.

d) Post-purchase behavior

The app should draw on consumer feedback system effectively. After coming to Hong Kong, it should retain such a system so that consumers can give restaurant and staff ratings or reviews after each consuming behavior. In addition, full-time customer service personnel should be recruited in Hong Kong to handle and maintain the relationship with consumers.

\subsection{Summary}

Based on the consumer need and buying process analysis, Meituan App ought to provide the relatively low service price to customers. Prior to introduce in Hong Kong market, Meituan should know the tastes preference of target customers, the preference cuisine is in connection with the selection of co-operate merchants. Satisfying the needs of consumer can develop a well rapport with customers and increase sales. Also, comprehending the characteristics of buying process in necessary.

\section{Consumer Evaluation Process Analysis}

In order to analyze the consumer evaluation for Meituan Take-out, the PEST analysis and SWOT analysis [8] are introduced as follows.

\subsection{PEST Analysis}

\subsubsection{Political Environment}

With the rapid development of e-commerce, the government has become more standardized and legalized in 
the management of e-commerce. Meanwhile, the government is actively developing more relevant laws and regulations to regulate e-commerce. Perfect political and legal environment can protect the development of Meituan.

\subsubsection{Economic Environment}

According to the data, the per capita GDP of Hong Kong in 2017 is more than $\$ 43,000$, and the per capita disposable income continues to grow, which is closed to the level of the high-income countries. At the same time, according to the latest data released by education department, the number of university students in Hong Kong is increasing year by year, and the number of mainland students is rising at the same time. In addition, the electronic commerce market develops rapidly, thus its foreground is bright. With the improvement of people's living standard and the change of living habits, e-commerce has a huge market. Besides, Gini Coefficient of Hong Kong is about 0.539 now, which is higher than many areas. This means a widening gap between rich and poor. And Meituan Take-out can hire local students who are familiar with routes and want to make money as part-time workers.

\subsubsection{Social-Cultural Environment}

With the rapid development of the Internet and the improvement of people's living standard, the e-commerce market has gradually matured and entered the golden stage of development. Due to the prevalence of Internet culture and the change of consumption concept, online shopping is not a strange thing for university students. University students are also a more active part of online ordering. Based on this kind of spending habits, Meituan take-out is able to meet the consumption demand of these students' online ordering.

\subsubsection{Technological Environment}

The fast development of Internet and intelligent technology has not only satisfied people's needs but also increased people's dependence on them [9]. Nowadays, Internet and intelligent technology become an indispensable part of people's daily life.

\subsection{SWOT Analysis}

\subsubsection{Strengths}

At first, group buying and take-out have synergies. From the point of view of the merchant, takeout does not occupy the real area of the restaurant, and the merchant will regard it as an extra income. This is an advantage for Meituan Take-out App.

Secondly, Meituan take-out is more convenient when comparing to eating at a nearby restaurant. It has the advantage of saving time and choosing variety. Next, up to 2017, Meituan has ranked first in mainland market share and experienced the fastest growth. Excellent word of mouth is conducive to the use of Meituan for students in HK, especially mainland students.

At last, Meituan has been developed in mainland China for many years, and its strength is relatively strong. It is relatively mature in business ability and technical ability. Its own publicity and planning team and talent resources also can facilitate its expansion in Hong Kong.

\subsubsection{Weaknesses}

Firstly, the self-built distribution team is still in its infancy. Due to unforeseen circumstances, delivery quality and speed cannot be guaranteed. Next, promotion channels and methods still need to be improved. In Mainland China, its propaganda leaflets are not widely distributed, and there are relatively few physical advertisements, which are mainly promoted online by Internet. If Meituan wants to enter the Hong Kong market, it needs to improve in this aspect. Thirdly, the use of network technology to "brush bills" would result in waste of resources. Fourthly, the service flow and design of APP need to be further optimized.

\subsubsection{Opportunities}

At first, it has large consumption groups and broad consumption market. It can meet people's "lazy" needs [10]. In other words, it has the ability to meet the needs of most students without going out. Secondly, the arrival of the Internet era is gradually changing the development trend of the catering market, from the traditional model to $\mathrm{O} 2 \mathrm{O}$ (customer-platform-merchant) model. With the increasing trend of Internet users yearly, the potential of target consumer market would further increase.

Next, with the popularization of smart phones and the promotion of mobile network [11], the difficulty in the development of $\mathrm{O} 2 \mathrm{O}$ market in catering industry has been greatly reduced, and the time for take-out has been matured. At last, with the continuous improvement of the information level of catering industry, the new technology can be used to grasp the user demand, improve the service quality and enrich the user experience.

\subsubsection{Threats}

Firstly, the competition in the industry is fierce, local take-out brands in Hong Kong and other mainland businesses that want to enter Hong Kong market are strong competitors. Secondly, it is difficult to absolutely occupy the market because of low user loyalty and lack of core competitiveness (users can't eat a take-away food for a long time).

Thirdly, with the largest proportion of student users, the students' consumption ability is relatively limited, so there is a little room for profit. Fourthly, the distribution cost for some businesses may be too large to accept. Lastly, due to the difficulty in ensuring the health of the catering industry, it is easy to produce litigation disputes. Sometimes disputes cannot be avoided.

\subsection{Summary of Market Research}

Considering about the PEST analysis of Meituan, the government pays more attention on standardized and legalized in the management of e-commerce. It will be helpful for Meituan to operate the online business. Besides, the HK government allowed the most popular payment apps from mainland China like WeChat Pay or Alipay, it means that the online payment become more convenient than credit card. 
Based on the SWOT analysis, the core team of Meituan has much experience of websites and business negotiation skills. Besides, as the top of take-out service app in mainland, Meituan has relatively high brand awareness. The positive word-of-mouth communication and peer influence will help Meituan introduce in Hong Kong. However, the self-built distribution team is still in its infancy, it will impact the efficiency of the employees to delivery. Besides, the costs of job training should be considered. Undoubtedly, the catering market will change because of the Internet applications and services become popular, that should be the chance for Meituan. And its experiences of operating the website in mainland would be useful to catch the loyal customers than other companies.

\section{Product Focus and Goal Setting}

\subsection{Market Segmentation}

Geographic segmentation: eight universities of Hong Kong. To arouse the interests of target customers by low delivery fees and promotion activities.

\subsection{Market Targeting}

Targeting: Hong Kong university students of from Mainland China. The goal is to enhance the service quality by emphasizing user experience and dealing with feedbacks.

\subsection{Market Positioning}

Positioning: convenient payment modes; low delivery fees; more cooperate merchants; self-allocated map on the platform. The goal is to grab the market shares and obtain potential customers by comprehending the consumers' mind-set.

\section{Recommendations for Marketing Actions}

In order to attract a large number of student groups in Hong Kong and promote the app to the whole market in later stage, the following suggestions should be noticed.

Initially, Meituan ought to reduce the human resource expenditure. It is generally known that the hefty price tag on labor costs in Hong Kong. It is suggested that Meituan is necessary to hire more local students as delivery guys and adopt shifting system, although Hong Kong is a bustling metropolis and well known as financial center in Asia and the GINI coefficient reached 0.539 that range between zero and one. For those students from low-income families, a part-time delivery job is attractive for them even though the wages and benefits are lower than other jobs. The high education level or any long-time training is not the prerequisite for addressing delivery service. Also, the local students are competent for such job because of the high degree of familiarity of local location and condition.

Secondly, based on the product life cycle [12], in the initial stage, Meituan take-out app will be stayed in the introductory stage. Hence, Meituan should demonstrate an appropriate marketing mix [13].

i. Product: provide service and warranty, such as delivery time, incubator sanitation and the interface design of APP;

ii. Price: lower than competitors;

iii. Place (channels): MTR, KMB; social media: Facebook, Instagram, Whatsapp, official account to promote interactivity;

iv. Promotion (Advertising): 1) sales promotion: attractive discounts and random reductions; 2) PR: examine public attitudes toward some promotion activities of app; executive campaigns to earn public understanding the app.

Thirdly, concerning the advertising, Meituan can ponder to adopt celebrity endorsement [14] to attract target customers. For instance, in the initial stage, Meituan can continue to invite famous young stars from Mainland China to attract mainland students. And the firm may consider to invite the famous local gastronome named Choi Lan and young local stars in later period. Still, Meituan should concern about product placement. For example, $\mathrm{QR}$ code on restaurant table, the printed advertisement on MTR and KMB.

Fourthly, although the quality and price is key for competition, customer loyalty [15] cannot be neglected as well. One of threats in SWOT analysis part is the low user loyalty and lack of competitiveness. Consumers are impossible to order takeout all the time, so a series of activities sponsored by Meituan can increase frequency to use the app when reinforcing the brand identity. From the RATER model [16], the service quality plays a key role in maintaining customer loyalty.

i. Responsiveness-The speed of order receiving should be as soon as possible;

ii. Assurance-The sanitation of food should be promised;

iii. Tangibles-The incubator sanitation; Staff should dress in Meituan uniform; Supporting system to record the flavor preference of loyal customers;

iv. Empathy-Except for interaction by social media with customers, Meituan have access to customers and then grab the comments of service provided by launching activities;

v. Reliability-The food are from cooperate merchants with good qualification.

Moreover, Meituan ought to adopt the relatively low delivery fee than other take-out applications at the beginning stage. In comparison with competitors, Meituan app has stronger financial competence. Its advanced management and operation mode can help the company run in Hong Kong.

Eventually, it is recommended that Meituan app ponder to cooperate with specific merchants. The tastes of cuisine can help people to find self-identity group. Meituan can utilize the preference cuisines ranking to choose co-operate merchants. The data is collected from 248 effective copies of total questionnaires (total: 300). Attached data would be helpful. 


\section{Conclusion}

This paper takes the Meituan App as the research object. Firstly, through a large number of market research, the opportunity and possibility of its entry into the Hong Kong market are established. Then, under the assumption that it will enter the new market in Hong Kong, the target customer group and clear product positioning are formulated. At the same time, this case study proposes a specific and comprehensive marketing promotion plan. In order to promote the efficient practice of the promotion plan, detailed operational procedures and corresponding theories are also described above. This paper aims to study whether the Meituan App can enter HK area and how it successfully enter the new market. It is hoped that this study can provide valuable guidance for Meituan APP and other similar companies in this industry.

\section{Appendix}

The questionnaire of this research:

Cuisine from: Sichuan (216/248) > Guangdong (208/248) > Hunan (88/248) > Jiangsu (60/248).

Questionnaire on the Prospects of Meituan Take-out App in Hong Kong's Universities

Thank you for participating. The Meituan Take-out App is an Internet ordering platform and the combination of O2O and selling industry. In the use of the Internet to provide take-out services, it contacts buyers and sellers. According to the popularity of take-away in mainland China, we have designed the following questions. Your answers will help us to understand the prospects of Meituan Take-out App in Hong Kong's market for college students from mainland. (Estimated: 300; Actual: 248)

Part 1:

1. Do you often eat out when you are busy with studies? A. Often 236 B. Not 12

2. Are you willing to share the table with others or queue? A. Yes 50 B. No 198

3. If the Meituan Take-out promotes the Hong Kong market, on the Health level (1 means hardly significant; 7 means extremely significant). $\quad 5.88$

To what extent does the health level affect your use of Meituan Take-out App?

4. Taste (1 means hardly significant; 7 means extremely significant). $\quad 5.76$

To what extent does the taste affect your use of this take-out App?

5. Service (1 means hardly significant; 7 means extremely significant). $\quad 5.43$

To what extent does the service affect your use of this take-out App?

6. Speed (1 means hardly significant; 7 means extremely significant). $\quad 6.46$

$\begin{array}{lllllllll}\text { To what extent does the speed of food delivery affect your use of this take-out App? } & 1 & 2 & 3 & 4 & 5 & 6 & 7\end{array}$

7. What is the most important factor that motivates you to use this App?

A. Health level 38 B. Taste 32 C. Service 5 D. Speed 173 E. Others (please point out):

8. What is your personal preference in the following four tastes? (According to your taste and preference: 1 indicates mostly preferred; 5 means hardly preferred).

1. Light food 58

2. Spicy and hot food 128

3. Salty food 49

4. Sour-sweet food 10

5. Others (please point out): 3

9. What is your personal preference in style of cooking? (According to your taste and preference: 1 indicates mostly preferred; 5 means hardly preferred).

1. Sichuan cuisine 216

2. Hunan cuisine 88

3. Shandong cuisine 27

4. Cantonese cuisine 208

5. Huizhou cuisine 39

6. Zhejiang cuisine 46

7. Jiangsu cuisine 60

8. Fujian cuisine 45

9. Others (please point out):

10 . What is the delivery time you can accept?

A.10-15 minutes 52 B.15-30 minutes 160 C.30-45 minutes 36 D.45-60 minutes E.60 minutes or more F. Others (please point out):

11. What is the service attitude of the take-away personnel you have contacted? ( 1 means worst attitude; 7 means best attitude.)

Service attitude of staff (6.24)

12. What is your score for this take-out app? ( 1 indicates the lowest; 7 means the highest)

Score (5.96)

13. Which point do you value mostly for this app? (1 means the lowest; 5 means the highest) 
1. Food safety 6.38

2. Delivery speed 6.64

3. Service quality 5.16

4. Benefit degree 4.22

5. App design 3.32

6. Cuisine variety 5.45

14. What do you think the role of this app in the catering industry's development?

A. Helps small stores to survive in competition.

B. Increases sales rapidly.

C. Helps new stores and remote stores increase their reputation.

D. Others (please point out):

15. How do you know this app?
A. Network publicity 20
B. Offline posters 104

C. Friends recommend 80

D. Others (please point out): 44

16. How many times do you eat take-out food a week?
A. Under 3 times 136
B. Between 3-7 times 60
C. Between 8-12 times 40
D. Above 12 times 12

17. What is your order method of food usually?
A. Telephone 158
B. Mobile platform/Internet 42
C. Request for other persons 40
D. Others (please point out):
42

18. What is the take-away price that you can accept?
A. Under 40 dollars 45
B. Between 40-80 dollars 126
C. Between 80-120 dollars 48
D. Between 120-160 dollars 20

E. Above 160 dollars 9

19. Which promotion method do you prefer?

A. Return money when reaching a certain amount 158

B. Give free drinks when reaching a certain amount 24

C. Give coupons when reaching a certain amount 45

D. Discount package 20

E. Others (please point out):

20. What is your advice for improving this app?

Part 2: (Personal Information)

1. Gender: Male 111 ; Female 137

2. Grade: A. Freshman 40 B. Sophomore $45 \quad$ C. Junior 31 D. Senior 8 E. Postgraduate and above 124

3. Living expenses/per month:

A. Under 3,000 HKD

C. Between 8,000 -13,000 HKD 188

B. Between 3,000 - 8,000 HKD 22

D. Between 13,000 -18,000 HKD 30

E. Above 18,000 HKD 8

4.Where are you from?

Beijing 15 Tianjin 4 Shanghai $\underline{4}$

Chongqing 5 Shaanxi_2_- Shanxi $\_5$ _ Guangdong $\_82$ Hebei _3__Anhui_ 8 Zhejiang _27_Liaoning _ 9 _Jilin_9 Heilongjiang $\overline{13}$ Jiangsu_ $\overline{5} \_$_ Fujian $\frac{3}{3}$ Jiangxi_2_Shandong_4_Henan_4 Hubei_3 - Hunan_9 Hainan_ 1 Sichuan $\overline{16}$ _ Guizhou Yunnan Gansu Qinghai_ Neinenggu_2_ Guangxi

4 Tibet
$\overline{\text { Xinjiang }}$ Ningxia

Consumer Marketing, 2014, Vol. 26 (1), pp. 14-28.

\section{References}

[1] Uriel Abulof, "Introduction: Why We Need Maslow in the Twenty-First Century,” Society, 2017, Vol. 54 (6), pp. 508-509.

[2] Elena Rasskazova, Tatiana Ivanova, Kennon Sheldon, "Comparing the effects of low-level and high-level worker need-satisfaction: A synthesis of the self-determination and Maslow need theories," Motivation and Emotion, 2016, Vol. 40 (4), pp. 541-555.

[3] Deqiang Zou, Liyin Jin, Yanqun He, Qian Xu, "The Effect of the Sense of Power on Chinese Consumers' Uniqueness-Seeking Behavior," Journal of International

[4] Gallayanee Starwind Yaoyuneyong, Wesley A. Pollitte, Jamye K. Foster, Leisa R. Flynn, "Virtual dressing room media, buying intention and mediation," Journal of Research in Interactive Marketing, 2018, Vol. 12 (1), pp. 125-144.

[5] Anonymous, "Emerging Company Profile; Meituan.com," Interfax: TMT China Weekly, 2011.

[6] Anil Kumar, Sachin Kumar Mangla, Sunil Luthra, Nripendra P. Rana, Yogesh K. Dwivedi, "Predicting changing pattern: building model for consumer decision making in digital market," Journal of Enterprise Information Management, 2018, Vol. 31 (5), pp. 674-703. 
[7] Chan, K., Youth and Consumption. City University of Hong Kong Press, Hong Kong, 2010.

[8] Solomon, Consumer Behavior: Buying, Having, and Being (11 ${ }^{\text {th }}$ Ed.), Pearson. 2015.

[9] Cecilia Zilima Mussa, "The Influence of Social Media on Consumer Behavior: An Empirical Study on Factors Influencing Consumer Purchase Intention in Malawi," University of International Business and Economics, Beijing, 2017.

[10] Thompson S.H. Teo, Yon Ding Yeong, "Assessing the consumer decision process in the digital marketplace," Omega, 2003, Vol. 31 (5), pp. 349-363.

[11] Alessandra Papetti, Andrea Capitanelli, Lorenzo Cavalieri, Silvia Ceccacci, Francesca Gullà, Michele Germani, "Consumers vs Internet of Things: A Systematic Evaluation Process to Drive Users in the Smart World," Procedia CIRP, 2016, Vol. 50, pp. 541-546.
[12] M. O’ Driscoll, “Design for manufacture," Journal of Materials, 2002.

[13] Liping LI, "Analysis Of The International Marketing Mix," Overseas English, 2013(23).

[14] Liz Hill, Terry O‘Sullivan, Foundation Marketing, Macro Environment; Distribution Channels, 2004.

[15] Valarie, Dwayne, Mary. (2013). Service Marketing: Integrating Customer Focus Across the Firm (6th Ed.).

[16] Jodi M. Casabianca, Brian W. Junker, Ricardo Nieto, "Mark A. Bond A Hierarchical Rater Model for Longitudinal Data," Multivariate Behavioral Research, 2017, Vol. 52 (5), pp. 576-592. 